\title{
Kauflächen-Veneer auf vorhandener VMK-Brücke
}

Der Filmpreis 2016 der Arbeitsgemeinschaft für Keramik in der Zahnheilkunde (AG Keramik) wird für ein 3-minütiges Video an Dr. Taskin Tuna, RWTH Universitätsklinikum Aachen, Abt. Zahnärztliche Prothetik und Biomaterialien, gehen. Der Preis für den Kurzfilm „Keramisches Table Top auf Brücke im Rahmen einer FullMouth Rehabilitation“ wird dem Gewinner auf dem 16. Keramiksymposium am 26. November 2016 in Hamburg übergeben. Die Laudatio wird Dr. Bernd Reiss, 1. Vorsitzender AG Keramik und Vorstandsmitglied der DGZMK, halten. Das prämierte Video „Keramisches Table Top auf Brücke“ der Session 2016 ist auf der Website der AG Keramik zu sehen: www.ag-keramik.de Menü Aktuelles/Videopreis Die Jury hat sich zu dieser Auszeichnung entschlossen, weil Tuna (früher Univ. Freiburg) mit seinem Videobeitrag ein ungewöhnliches, nicht alltägliches Thema gewählt hatte. Erstmalig wurde die Verblendung einer mehrgliedrigen VMK-Brücke im Molarenbereich zwecks Ebenenkorrektur mit einem vollkeramischen Table Top restauriert. Der basale Anteil der Brücke blieb dabei erhalten. Eine ganze Reihe praktischer Tipps, die systematisch konsequente Durchführung, die hohe Qualität der Darstellung und die didaktische

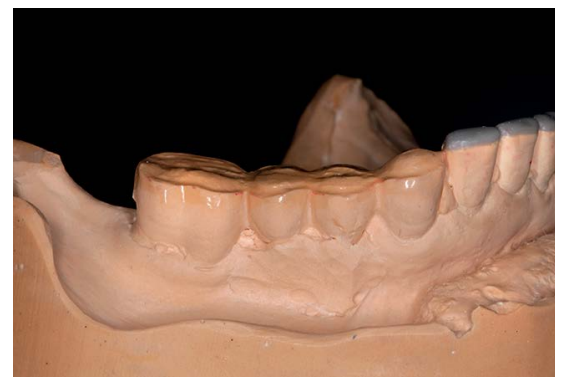

Klarheit wurden als preiswürdig eingestuft, da diese auch auf weniger exotische Ausgangssituationen anzuwenden sind. Ausgangssituation war ein insuffizient versorgtes Gebiss mit hängender Ebene im 1. und 4. Quadranten. Auf Wunsch des Patienten sollte die bestehende, implantatgetragene VMK-Brücke regio 43-46 trotz Furkationgrad 3 bei guter Mundhygiene erhalten und weitere augmentative Maßnahmen im Rahmen einer Implantattherapie verhindert werden. Daraus entwickelte sich das Behandlungskonzept, lediglich die Okklusalebene mit einem keramischen Table Top minimalinvasiv zu korrigieren.

Nach einer Pressemitteilung der AG Keramik, Ettlingen info@ag-keramik.de 